\title{
A SMARTPHONE GAME TO PROMOTE SELF-LEARNING IN CHEMISTRY
}

\author{
Krittiya Saksrisathaporn and Patcharaphon Sribunthankul \\ College of Arts, Media and Technology, Chiang Mai University \\ Chiang Mai 50200, Thailand
}

\begin{abstract}
In Thailand, for most students in high school, remembering the periodic table is an important part of learning chemistry and to pass a university entrance examination in their scientific program. As many students live in the bigger city areas in Thailand, most of them encounter the same problems such as limitations on lesson time, and their involvement in a wide range of high school and extra-curricular activities which reduces the student's study time. Self-learning is one method that has been proven to be effective, convenient, and fast. Presently, there are few effective resources for students to enjoy self-learning. Game-based learning is one of the approaches that has been suggested, but most of them are too difficult to learn how to play, not attractive and not challenging. In this study, a smartphone-based learning game was designed and developed with better level design, including color emotional theory, and quality graphic design to provide a better user experience. The game consists of three stages, and at each stage, a mini-puzzle game is presented which plays a different style with different goals to be achieved. The first stage of the game is to remember the names and symbols of elements. The second stage is to remember the group and period, while the third stage allows students to apply elements to form chemical compounds. The results from an evaluation showed that $96 \%$ of 102 students, enjoyed playing the game, $87 \%$ said the game was challenging, and $94 \%$ remembered at least 3 elements more than before playing. The satisfaction questionnaire demonstrated the benefits of the game-based learning system on self-learning in chemistry.
\end{abstract}

\section{KEYWORDS}

Game-Based Learning, Serious Game, Chemistry, Periodic Table, Smartphone Game, Android

\section{INTRODUCTION}

The Periodic Table is one of the topics listed in the science program course for high school and university students in Thailand and is part of the compulsory education curriculum of Thailand. It is considered very important for the Thailand university entrance examination in the scientific program. Moreover, it is a subject that is included in other higher education courses such as Science of Chemistry, Chemical Engineering, Pharmacy, etc. Combined with other lessons that students must learn to prepare themselves for future lessons within the subject make it hard for them to remember. Due to many students in high school living in bigger cities, most of them encounter the same problems with limits on lesson time and being overloaded with additional work from teachers. The student-teacher ratio is also often too high. Furthermore, student's study time is reduced significantly because of the number of activities high school or university students are expected to participate in. Therefore, studying a periodic table, and trying to remember all the elements becomes boring and repetitive, more so when trying to remember from textbooks. If students are unable to learn and remember all the important details of the main topics, essential elements, the periodic table, and chemical compounds, it will continually affect their future learning in chemistry, making it difficult to continue learning, and pass yearly university entrance exams. However, game-based learning has been used as a tool for centuries and is much more attractive and interesting, and results in active learning rather than passive learning through textbooks and theory (Saksrisathaporn \& Maneewan, 2012).

Currently, there are only a few other resources that can help students learn chemistry by themselves. Game-based learning is very close in meaning to a serious game, and a serious game has become a hot topic in training and education as shown in the abundant academic research since 2012 (Guillen, V and Aleson-Carbonell, M, 2012). Mostly, the digital game-based learning available in the market does not focus on graphics or level designs. For example, the Kagaku Chemistry Periodic Table of Elements only displays the 
table on a scrollable screen and includes buttons for explanation and a search window (Freewarepocketpc, 2017). Also, mobile devices have become widely popular devices because they are easy to use, portable and can support a variety of applications.

In this study, a smartphone game named "Chemistry Pop" was designed and developed. The proposed game was designed to help students to learn chemistry with more interest and enthusiasm. This mobile game aims to support science students to learn and remember the essential elements in the periodic table with more interest and enthusiasm and to increase the resources that may help aid students in self-studying. The game is designed with colorful graphics.

The students can play a game and remember the essential elements in the periodic table at the same time. They can also continue growing and improving these memories at a higher level of education. The game consists of three stages, each stage plays differently and has different goals. The first stage is to support the student to remember the names and symbols of elements from the periodic table. The gameplay of this stage is adapted from a "Bubble Shooter" game. The single-player video game Bubble Shooter probably became famous as it is very intuitive and easy to learn. It is a mix of "Tetris" and "Connect Four" (Shooter-bubble (2019). This creates a game addiction feeling similar to the Candy Crush Saga game (Chen, C., 2016). The aim of Bubble Shooter is to collect as many points as possible. To get points you need to destroy the colored bubbles. For the Chemistry pop game, the player must connect and group at least three bubbles of the same property to collect them according to the goal, which is given at each level. Players must shoot the bubbles with given symbols to collect and clear the goal, which uses the elements' names. To pass each level, players must clear all progress bars within the time and moves limits. The second stage is to help students remember the group and period of elements from the periodic table. The bubbles with element symbols will be released randomly and will always be moving. Players must use their fingers to touch, hold and drag to form a circle around bubbles with the same property and need at least three bubbles to collect them and clear the goal, which would be the group or period numbers. The last stage is to help students remember the 5-chemical compounds. The bubbles will be collected if the connection of bubbles is in accordance with a chemical compound. To pass each level, players must clear all progress bars within the time limit. The prototype game was tested and evaluated with a sample student population.

The remainder of this study is structured as follows: Section 2 describes the background and related work. Section 3 presents a flow of the research methodology. Section 4 presents the game design and development. Game testing and evaluation are discussed in Section 5. Lastly, conclusions and future perspectives are discussed in Section 6.

\section{BACKGROUND AND RELATED WORK}

\subsection{Element and Periodic Table}

The International Union of Pure and Applied Chemistry (IUPAC) have announced that there are 118 elements, and each element has its own name and symbol, and they are placed on a table by dividing the table into 16 groups and seven periods (IUPAC, 2017). The position of elements on the table is according to the property of each element, which is another important topic for students to learn in chemistry. In addition, the chemical compound is also an important topic because it forms the basic knowledge needed for almost all future lessons within the chemistry subject. Each compound also has its own formula and unique name.

\subsection{Game-based Learning and Serious Game}

Currently, there are many types of games such as board games, card games, video games, and digital game. These games can be type constructed as an entertainment game, game-based learning, simulation-based games, serious games, etc. According to Lorenz, 2005 (Lorenz et al., 2015) people who play video games have better memory and strategic planning capabilities compared to those who do not play video games. They also have a larger quantity of gray matter, which is the part that sends information to the brain and is an important part of the central nervous system. In 2014, Andre proposed a method of a serious game to test the "Clean world" game. The learning of games occurs within multiple mechanisms, such as mini-games, puzzles, and quizzes played in parallel to the main game environment (Andre et al., 2014). 
Game-based learning has been identified as an effective approach to making learning activities playful and engaging (Klopfer et al., 2009). According to Kammardsiri, game-based learning or serious games is one of the assistant systems to improve learning for students in knowledge acquisition, training and learning skills (Kammardsiri et al., 2017). Additionally, the games provide feedback to the student, great improve their motivation and provide enjoy learning outcomes. In 2016, Hsiao and Chen developed a gesture interactive game-based learning (GIGL) approach for improving pre-school children (Hsiao and Chen, 2016). In 2017. Fujimoto presented JobStar Online: Game-Based Learning on Smartphones to Promote Youth Career Education. This game is designed to help youth develop a positive attitude towards career development and prepare for emerging opportunities (Fujimoto et al., 2017). To design digital game-based learning for high school students, many principles are considered to develop these mobile-based educational games. Recently, many researchers focused on game-based learning or serious games to enhance the learning skills of students. As, interactive games can bring intuitive content directly to students through their devices.

Some educational games are traditional games (puzzles, card games, etc.) (Franco et al., 2016). However, most education game-based learning is simple in level design and graphic design. Some of them are puzzle games designed for on PCs or websites (Periodic table Game, 2018). In 2016, Chairs! a mobile game for Organic Chemistry Students to Learn the Ring Flip of Cyclohexane by drawing the Ring Flip of Cyclohexane on the smart phone was developed by Winter (Winter et al, 2016). Also, the simple graphics game named 2048 Periodic Elements is an interesting and very addictive puzzle Game (Google, 2017). Additionally, Chemical Bonding is a learning unit that incorporates an elicitation strategy in the context of chemical bonding for high school students (Rompayom, 2010). This application is run on a mobile device with good quality graphics and plays like a game but is more focused on content than a gameplay feeling.

\subsection{A Smartphone Games}

According to Granter's global smartphone and tablet survey in 2015, Android has the largest market of smartphones. Besides, InMobi, one of the largest advertising networks in the world, found that Thailand has the highest usage of Android applications through collecting information from the impression count of advertisements. On average, people spend 230 minutes on smartphones daily. According to released data from Thailand's National Statistical Office (NSO) in January 2017, more than $90 \%$ of internet users in the country go online via smartphones. More than $50 \%$ of mobile phone users use smartphones and it is expected that the percentage will grow (NSO, 2017). In this study, the Chemistry Pop game is made by Unity. The Unity software is a game engine for developing 2-dimensional (2D) and 3-dimensional (3D) games, which can work on two platforms, Windows and OSX. This game-engine can be exported or deployed to 17 different platforms, for example, Windows, Mac, Web, Android, iOS, consoles, etc. The popular 2D game "Gardenarium" by Paloma (2017) and Kyler (2005) with the support of KO-OP (Kiili, 2005) was also made by Unity software.

\section{RESEARCH METHODOLOGY}

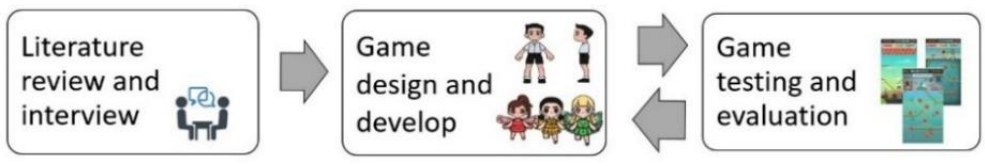

Figure 1. Research methodology

As shown in Figure 1, the research started with a literature review and interviews with four teachers who teach science and Chemistry at Montfort College, a high school in Chiang Mai, Thailand. As mentioned in the introduction section, some problems were listed. Game-based learning was one of the approaches suggested and chemistry was one of the topics that were selected for content to create a prototype game. The smartphone game was designed and developed using Unity software. In this study, a puzzle game was designed and developed. The game is divided into 3 stages creating mini games. The game was tested and evaluated by 102 players (students in the science program). 


\section{GAME DESIGN AND DEVELOPMENT}

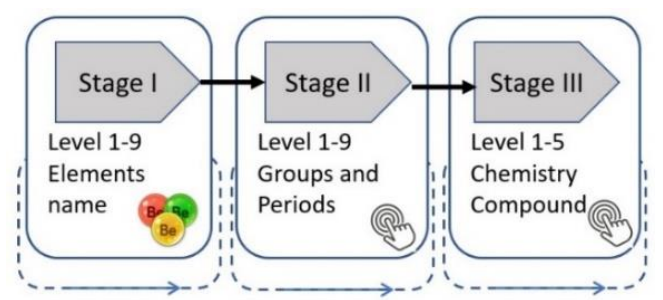

Figure 2. Diagram of 3 stages

As shown in Figure 2, the main game is included with three mini-games in three stages. The first one (stage I) is like a Bubble Shooter game to remember the element's name. The second one (stage II) is a group and period mini game. To play the game, the player must touch, hold and drag their finger to create an area around bubbles of the same group or period to capture them on the screen of the smartphone. The last one (stage III) is a Chemical compound mini game, the gameplay is similar to stage II. The player must collect the compounds according to the goal given. Each game stage is divided into nine levels and each level can be composed of several learning mechanisms. For gameplay, game stage III is influenced by stage II and stage II is influenced by stage I, when the player can remember the element's name and can put them in the right group and period in stage II. The number of atoms depends on groups and periods, which will be the initial compound lesson of Chemistry in stage III. These learning mechanisms aim to embed the knowledge learnt. To design and develop the game with playable quality graphics, it has a detailed graphic design with color sensation. As Figure 3 shows, student name L is a player at all stages, Atom is the fairy of stage I, Group is the fairy of stage II and Compound is the fairy of stage III.

\subsection{Character and Scene Design}

The short story of the Chemistry Pop game was written. On Friday afternoon in a high school, a student name "L" let all elements in the periodic table escape in a Chemistry laboratory. Fairies "Atom", "Group" and "Compound" ask for responsibility to get them back, so L needs to go and find all escaped elements by playing 3 stages of the game. The game scenes are designed differently regarding time and level design, and the level of difficulty depends on each level in each stage.

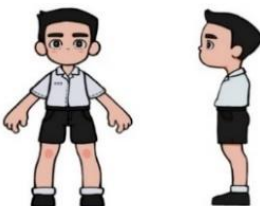

(a) Student L

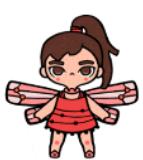

(b) Fairy Atom

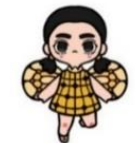

(c) Fairy Group

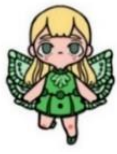

(d) Fairy Compound

Figure 3. Character design

To design and develop a mobile game that has a fine graphic design and provides a playable user experience design. The scene design for stage I and II are different regarding time (Morning, afternoon and night as shown in Figures 4, 5, 6, and 7), and the Kitchen and bathroom scene is designed for stage III (Figures 8 and 9). According to Supawan (Supawan, 2009), colors invoke different emotions thus colors selected in the game design consist of the color red to give sensational feelings, excitement, and challenges. The emerald color provides encouragement, serenity, peace, reduces fatigue and tension, reduces feelings of loneliness but increases communication power. Yellow is the color of happiness, vitality, celebration, involves wisdom, ideas, creativity and encourages optimism. Orange is the color of joy, freedom and provides a liberating feeling. Violet is the color of care and comforting, to help calm the mind and endure, to balance the mind, to recover from depression or sadness, to reduce desperate feelings, and increase cheerfulness and relaxation. Finally, brown is the color of the earth and provides a stable feeling. 


\subsection{Game Level Design}

In this study, the first 20 elements of the periodic table were selected in all stages of the game. They are Hydrogen, Helium, Lithium, Beryllium, Boron, Carbon, Nitrogen, Oxygen, Fluorine, Neon, Sodium, Magnesium, Aluminum, Silicon, Phosphorus, Sulphur, Chlorine, Argon, Potassium, Calcium, and Titanium, but Titanium is only added to the last stage. As shown in Figure 2, stage I and II consists of 9 levels and stage III consist of 5 levels, each stage plays differently and has different goals. The player needs to pass the first until the last stage by unlocking each level. To pass each level, players must clear all bubbles within the time limit. The first level of each stage is easy and will get harder and harder with the new random element from the previous level until the last level. In each level, the player needs to remember the element from the level before they can pass each level until the last level.

The structure of the game level design of all stages is detailed in Table. 1. For example, in stage I, level 1, the possible bubbles are Hydrogen $(\mathrm{H})$ or Helium $(\mathrm{He})$ (total= 2 type elements), In stage I, level 2 the possible bubbles are Lithium $(\mathrm{Li})$ and random element from level 1 ( $\mathrm{H}$ or $\mathrm{He})$ (total=2 type elements). The goal of the first stage is to remember the names and symbols of elements from the periodic table. The goal of the second stage is to remember the group and period of elements. The goal of the last stage is to remember the formulas of the 5-chemical compound which consists of laundry detergent $(\mathrm{NaOCl})$, salt $(\mathrm{NaCl})$, bathroom cleaning liquid $(\mathrm{HCl})$, liquid paper $\left(\mathrm{TiO}_{2}+\mathrm{H}_{2} \mathrm{O}\right)$, and toothpaste $\left(\mathrm{NaF}+\mathrm{Al}_{2} \mathrm{O}_{3}\right)$.

Table 1. Level design of game stage I II and III

\begin{tabular}{|c|c|c|c|c|c|c|c|c|c|c|c|c|c|c|c|c|c|c|c|c|c|c|c|}
\hline Stage & Level (lv) & $I$ & $\stackrel{0}{ \pm}$ & 3 & ๑ & 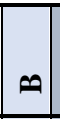 & 0 & $\mathbf{Z}$ & 0 & I & $\ddot{z}$ & $\underset{\mathbf{Z}}{\mathbf{Z}}$ & $\sum^{\infty}$ & « & $\bar{n}$ & $\theta$ & $\infty$ & $\bar{U}$ & 4 & 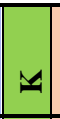 & שే & $\ddot{F}$ & $\begin{array}{l}\text { Random } \\
\text { elements } \\
\text { lv (num) }\end{array}$ \\
\hline \multirow{9}{*}{1} & 1 & 1 & 1 & & & & & & & & & & & & & & & & & & & & \\
\hline & 2 & & & 1 & & & & & & & & & & & & & & & & & & & $1(2), 2(1)$ \\
\hline & 3 & & & & 1 & 1 & & & & & & & & & & & & & & & & & $1(1), 2(1)$ \\
\hline & 4 & & & & & & 1 & & & & & & & & & & & & & & & & $2(2), 3(2)$ \\
\hline & 5 & & & & & & & 1 & & & & & & & & & & & & & & & $3(2), 4(2)$ \\
\hline & 6 & & & & & & & & 1 & 1 & & & & & & & & & & & & & $5(2)$ \\
\hline & 7 & & & & & & & & & & 1 & 1 & & & & & & & & & & & $6(2)$ \\
\hline & 8 & & & & & & & & & & & & 1 & & & & & & & & & & $6(2), 7(2)$ \\
\hline & 9 & & & & & & & & & & & & & 1 & & & & & & & & & $7(2), 8(2)$ \\
\hline \multirow{9}{*}{2} & 1 & 1 & 1 & & & & & & & & & & & & & & & & & & & & \\
\hline & 2 & & & 1 & 1 & & & & & & & 1 & 1 & & & & & & & & & & $1(1)$ \\
\hline & 3 & & & & & 1 & & & & & & & & 1 & & & & & & & & & $1(1), 2(2)$ \\
\hline & 4 & & & & & & 1 & & & & & & & & 1 & & & & & & & & $1(1), 2(3)$ \\
\hline & 5 & & & & & & & 1 & & & & & & & & 1 & & & & & & & $1(1), 4(2)$ \\
\hline & 6 & & & & & & & & 1 & & & & & & & & 1 & & & & & & $1(1), 5(2)$ \\
\hline & 7 & & & & & & & & & 1 & & & & & & & & 1 & & & & & $1(1), 6(2)$ \\
\hline & 8 & & & & & & & & & & 1 & & & & & & & & 1 & & & & $1(1), 7(2)$ \\
\hline & 9 & & & & & & & & & & & & & & & & & & & 1 & 1 & & $1(1), 2(1), 3(1)$ \\
\hline \multirow{5}{*}{3} & $\mathrm{NaOCl}$ & & & & & & & & 1 & & & 1 & & & & & & 1 & & & & & \\
\hline & $\mathrm{NaCl}$ & & & & & & & & & & & & & & & & & & & & & & \\
\hline & $\mathrm{HCl}$ & 1 & & & & & & & & & & & & & & & & 1 & & & & & \\
\hline & $\mathrm{TiO}_{2}+\mathrm{H}_{2} \mathrm{O}$ & 2 & & & & & & & 3 & & & & & & & & & & & & & 1 & \\
\hline & $\mathrm{NaF}+\mathrm{Al}_{2} \mathrm{O}_{3}$ & & & & & & & & 3 & 1 & & 1 & & 2 & & & & & & & & & \\
\hline
\end{tabular}

Remark: Group IA: H, Li, Na, K, IIA: Be, Mg, Ca, IIIA: B, Al, IVA:C, Si, VA: N, P, VIA:O, S, VIIA:F, CI and VIIIA: $\mathrm{He}, \mathrm{Ne}, \mathrm{Ar}$

\subsection{Gameplay}

For gameplay stage I as presented in Figure 4, the player must shoot the given bubble, which will be at the middle bottom of the screen, to hit the rows of bubbles at the top of the screen. The player must shoot the bubbles with the same element symbol (not the same color as the original bubble shooter game) to connect to 
each other at least three bubbles to make them pop. The goal is for the players to collect the elements according to the progress bars at the top of the screen, under the score, which will have the complete elements' names on them by popping bubbles with the symbol of the given names. To pass each level, players must clear all bubbles within the time or moves limits. The game will be over if the player cannot reach the goal within the time or moves given. If the last row of bubbles at the top touches the limit line, the game of that level will be over instantly.

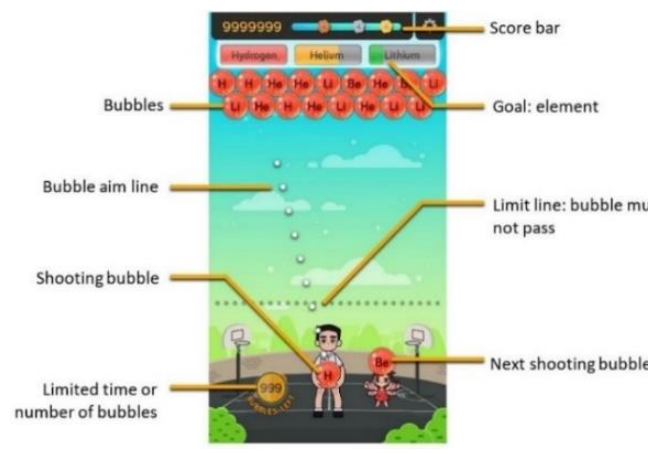

Figure 4. Screenshot of gameplay stage I
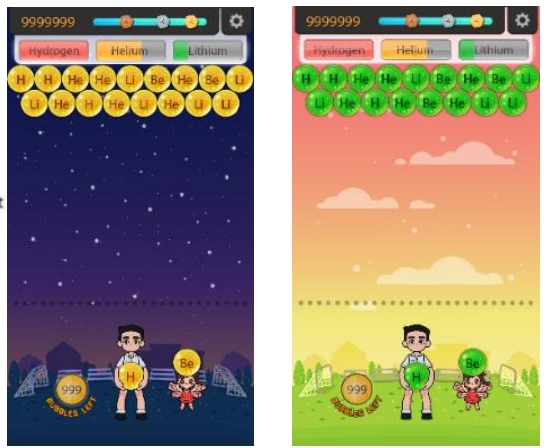

Figure 5. Basketball court scene for stage I

For gameplay stage II as presented in Figure 6, the bubbles will be released in random spots of the screen and will always be floating around. The player must touch, hold and drag their finger to create an area around bubbles of the same group or period to capture them. If it is successful, the bubbles are gathered into a bigger bubble. The goal is for the players to capture at least three bubbles of the same property according to the goal given in each level which will give the group or period number to collect them. If the player is unable to group at least three bubbles in a certain time, the big bubble will reduce back to its original form. To pass each level, players must fill all progress bars given (in this case, they are group 1A, 2B and period 1) within the time limit. The game will be over if the player cannot reach the goal within the time given.

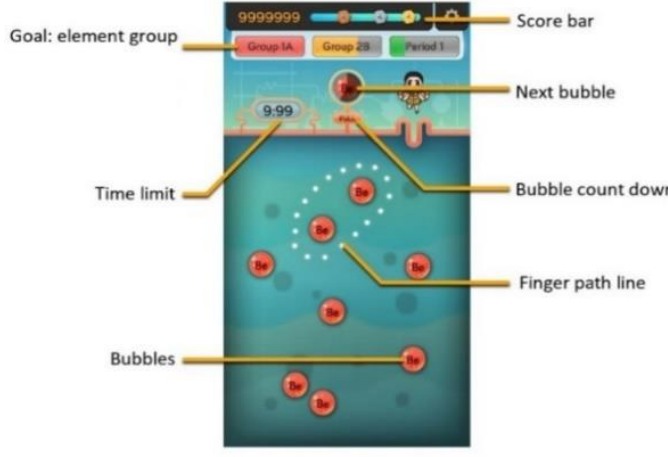

Figure 6. Screenshot of gameplay stage II
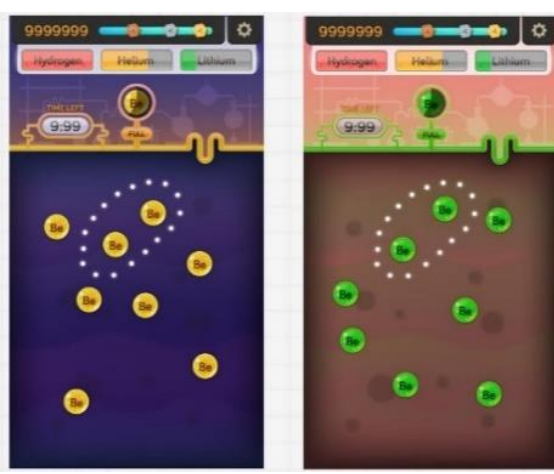

Figure 7. Inside a box scene for stage II

For gameplay stage III as presented in Figure 8. The player must draw around a pair of bubbles to form a connection of a chemical compound on the screen and reach the goal within the time given. Players can add more bubbles to that connection by circling one of the bubbles from the connection to a floating bubble. Also, the player can cut the connection by using their finger to touch, hold and drag a line to cut the connection line between the connected bubbles. The bubbles will be collected if the connection of bubbles is in accordance with a chemical compound. The goal is for the players to collect the compounds according to the goal given, which is the name of the chemical compounds. If the connected bubbles form a compound but it is not the goal the bubble will still be collected as score instead of progress. 


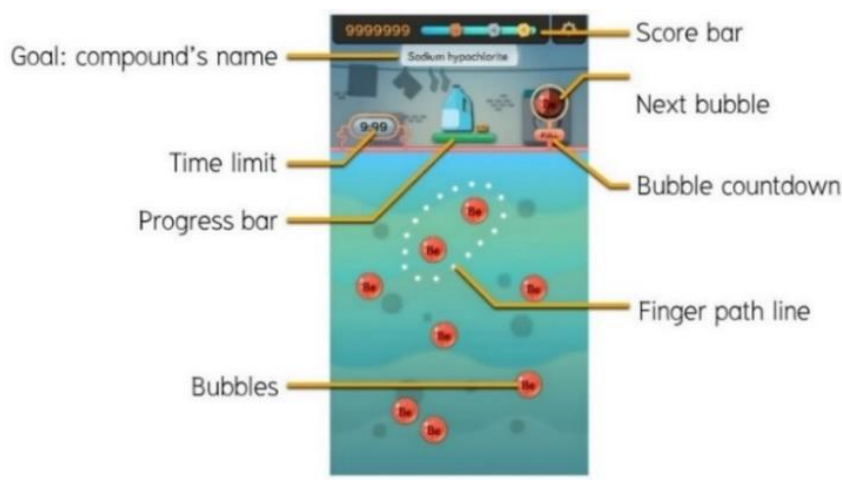

Figure 8. Screenshot of gameplay stage III

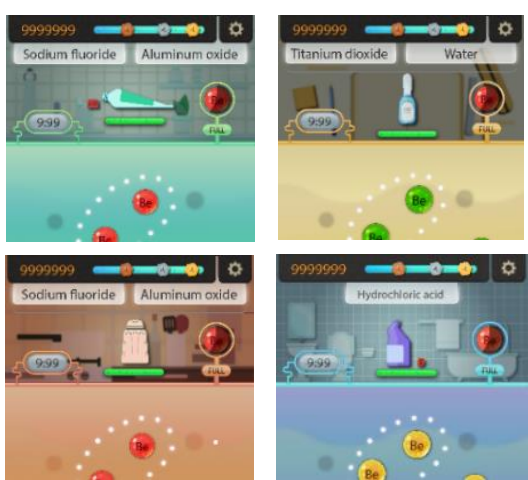

Figure 9. In the room scenes for stage III

\section{EVALUATION}

The research was conducted by testing the game with the sample group. According to a survey of 102 students in a science program, $63.8 \%$ male, $36.2 \%$ female, it was found that more than $90 \%$ liked to play computer games and more than $85 \%$ had played games on smartphones. Most of the sample groups have never experienced using game-based learning before. Moreover, there are more than $80 \%$ of the sample group that has experience in playing Bubble Shooter games, $96 \%$ enjoyed playing the Chemistry Pop game, $87 \%$ said the game is challenging. From inquiring about the knowledge of the periodic table, the survey found that $92.5 \%$ of the sample group had studied about the periodic table. In detail about learning techniques, $43 \%$ used reading memorization techniques, $20 \%$ used music with lyrics about the periodic table to help with memorizing it, $37 \%$ use other methods such as images, poems, etc., There are up to $82.5 \%$ of the sample that cannot remember the number of elements in the periodic table and up to 55\% that cannot remember symbols and names of the first 20 elements in the periodic table. After playing, $96 \%$ of 102 students, enjoyed playing the game, $87 \%$ said the game was challenging, and 94\% remembered at least 3 elements more than before playing.

Assessment of player satisfaction from the sample group after they played the game shows that the opinions of most samples say that the game is interesting and easy to play, the game can help the user to easily memorize, not as boring as normal reading memorization and can motivate the user to be interested in learning. Moreover, users also like the look of the game including characters and scenes. However, some participants found that the prototype game had some application errors occur that should be fixed. Some of them want the game to be further developed. With comments to add and edit certain functions to make the game more complete, including adding a system for introducing new elements that players will encounter in the game, adding extra items or extra points, improving animations of characters in the game to be able to show the emotions differently according to the situation, fixing the reviving duration portion for playing the game, and changing bubble speed.

\section{CONCLUSIONS AND FUTURE PERSPECTIVE}

This paper has presented the design and development of a smartphone game, a smartphone game to promote self-learning in chemistry, named Chemistry Pop. The game aims to apply the chemistry content of essential element names, the periodic table and chemical compound to players in a more fun and easier way to learn using gaming experiences like bubble shooter games, etc. This game-based learning consists of three stages with the content divided into stage I, learning element names, stage II, learning groups and periods, and stage III, learning chemical compounds. In addition, the user interface is developed and designed for easy usage, with quality graphics details similar to entertainment mobile applications. It allows the player to enjoy learning in a challenging environment without getting bored. The prototype game testing based on student needs proved the effectiveness and importance of game-based learning with education players. The survey results revealed that the players gained more knowledge of elementary chemistry than before playing. 
The research perspectives can be done by adding more elements from the periodic table into all three stages and applying other lessons to the game. To compare the game with other approaches used for science subjects. Finally, the Chemistry Pop game can be further developed and enhanced to meet the requirements of the general player and be adapted for a commercial game.

\section{REFERENCES}

Andre F. S. Barbosa. et al, 2014. A New Methodology of Design and Development of Serious Games, International Journal of Computer Games Technology, Article ID 817167, 8 pages.

Chen, C., and Leung, L, 2016. Are you addicted to Candy Crush Saga? An exploratory study linking psychological factors to mobile social game addiction. Telematics and Informatics, Vol 33, No. 4, pp 1155-1166.

Franco-Mariscal, A. J.et al, 2016. A Game-Based Approach to Learning the Idea of Chemical Elements and Their Periodic Classification. Journal of Chemical Education, Vol 93, No 7, pp 1173-1190.

Freewarepocketpc., 2017. Kagaku Chemistry Periodic Table of Elements, Retrieved from http://www.freewarepocketpc.net/ppc-download-kagaku-chemistry-periodic-table-of-elements.html

Fujimoto, T. et al, 2017. JobStar Online: Game-Based Learning on Smartphones to Promote Youth Career Education. Proceedings of the 25th International Conference on Computers in Education. New Zealand: Asia-Pacific Society for Computers in Education

Google Play., 2017. 2048 Periodic Elements Game. Retrieved from https://play.google.com/store/apps/details?id=com.game.chemistry.

Guillén-Nieto, V., and Aleson-Carbonell, M, 2012. Serious games and learning effectiveness: The case of It's a Deal! Computers and Education, Vol 58, No. 1, pp 435-448.

Hsiao, H.S. and Chen, J.C., 2016. Using a gesture interactive game-based learning approach to improve preschool children's learning performance and motor skills, Computers \& Education, No.95, pp.151-162.

IUPAC, 2017. The International Union of Pure and Applied Chemistry Retrieved from https://iupac.org/.

Kamnardsiri, T. et al, 2017. Designing of a game-based learning framework for training pharmacy students. The 11th European Conference on Games Based Learning, ECGBL 2017, pp. 301-310.

Kiili, K. ,2005. Digital game-based learning: Towards an experiential gaming model. The Internet and higher education, Vol 8, No. 1, pp. 13-24.

Klopfer, E. et al., 2009. Moving Learning Games Forward: Obstacles, Opportunities and Openness. MIT The Education Arcade. Cambridge, MA.

Lorenz, R. C.et al, 2015. Video game training and the reward system. Frontiers in human neuroscience, No.9, pp 40.

NSO, 2017. Thailand National Statistical Office. Retrieved from http://www.nso.go.th.

Paloma, 2017. Trippy, Dreamy Exploration: Gardenarium Released, Retrieved from https://www.rockpapershotgun.com/2015/04/03/gardenarium-exploration-game-released.

Periodic table Game. 2018, Retrieved from https://www.funbrain.com/games/periodic-table-game.

Rompayom, P., 2010. Development of Chemical Bonding learning units incorporated with elicitation strategy for high school students. Srinakharinwirot University, Thailand

Saksrisathaporn, K. and Maneewan, T.,2012. A Web-based Traveling Game for the Preservation of Lanna Culture. 2012 IEEE Fourth International Conference on Digital Game and Intelligent Toy Enhanced Learning, Takamatsu, Japan, pp. 52-56.

Shooter-bubble, 2019. Retrieved from https://www.shooter-bubble.com/

Supawan P., 2009. Color and emotion. Journal of Rajamangala Pitsanulok. Vol 3, Issue 11.

Winter, J.et al, 2016. Chairs! A Mobile Game for Organic Chemistry Students To Learn the Ring Flip of Cyclohexane. Journal of Chemical Education, v93, n9, pp.1657-1659. 